\title{
The Properties of Convex Function and its Application
}

\author{
Lele Fan, ${ }^{\mathrm{a}}$ Yixian Lao ${ }^{\mathrm{b}}$, Wusheng Wang ${ }^{\mathrm{c}}$ \\ School of Mathematics and Statistics, Hechi University, Guangxi, Yizhou 546300, P. R. China \\ afanlele262@sohu.com, b741679896@qq.com, cwang4896@126.com
}

Keywords: Convex function; Hadamard inequality; Proving inequality; Astringency.

\begin{abstract}
Convex function is a kind of very important function. In the proving inequality, optimization theory, operations research, control theory and other fields of applied mathematics, convex function has many applications. This paper introduces the definition and properties of convex function, and Hadamard inequality; then discusses applications of convex function in proving inequalities and verifying astringency of series.
\end{abstract}

\section{Introduction}

Convex function is an important concept in mathematical analysis, which relates to the proof of many mathematical propositions. In higher mathematics, we frequently encountered this kind of special function when we discuss properties of functions by derivative. Convex function has very practical value in many practical applied problems. Many authors [1-9] studied properties and applications of convex function.

Definition (see [1]) Suppose that $f(x)$ be a function defined on interval I. If the following relation $f\left(t x_{1}+(1-t) x_{2}\right) \leq t f\left(x_{1}\right)+(1-t) f\left(x_{2}\right), \forall x_{1}, x_{2} \in I, t \in(0,1)$,

holds. Then, $f(x)$ is called a convex function on interval $\boldsymbol{I}$. If the inequality (1) is the strict inequality, Then $f(x)$ is called a strict convex function on interval $\boldsymbol{I}$.

Lemma 1 (see [1]) If $f(x)$ is a differential function defined on the closed interval[ $[a, b]$. Then $f(x)$ is a convex function on the closed interval $[a, b]$ when and only when

$f^{\prime \prime}(x) \geq 0, x \in(a, b)$.

$f(x)$ is a strict convex function when and only when

$f^{\prime \prime}(x)>0, x \in(a, b)$.

Lemma 2 (see [1]) If $f(x)$ is differential on the closed interval[$[a, b]$, then the following statement are equivalence

$f(x)$ is a convex function on interval $[a, b]$;

$f^{\prime}(x)$ is a increasing function on interval $[a, b]$;

For $\forall x_{1}, x_{2} \in[a, b]$, the inequality

$f\left(x_{2}\right) \geq f\left(x_{1}\right)+f^{\prime}\left(x_{1}\right)\left(x_{2}-x_{1}\right)$

holds.

Lemma 3 (see [2]) If $f(x)$ is convex function on the closed interval[ $a, b]$, then the following inequality

$\varphi\left(\frac{a+b}{2}\right) \leq \frac{1}{b-a} \int_{a}^{b} \varphi(x) d x \leq \frac{\varphi(a)+\varphi(b)}{2}$

holds.

This paper firstly introduces the definition and properties of convex function, and Hadamard inequality; then discusses applications of convex function in proving inequalities and verifying astringency of series. 


\section{Application of convex function}

The convexity of convex function and its definition is established on the bases of inequalities. This fact makes the convex function to be an important tool in proving inequality.

Example 1 (see [2]) Suppose that $\varphi(x)$ is a continuous function on interval[0,a], and $f(y)$ is twice differentiable with $f^{\prime \prime}(x) \geq 0$, then

$$
\frac{1}{a} \int_{0}^{a} f(\varphi(t)) d t \geq f\left(\frac{1}{a} \int_{0}^{a} \varphi(t) d t\right) .
$$

Proof. Let $b=\frac{1}{a} \int_{0}^{a} \varphi(t) d t$, then (6) can be rewritten

$\frac{1}{a} \int_{0}^{a} f(\varphi(t)) d t \geq a f(b)$.

Since $f^{\prime \prime}(x) \geq 0, x \in(-\infty,+\infty)$, by Lemma 1 and Lemma 2, we have $f(x) \geq f(b)+f^{\prime}(b)(x-b)$, thus

$f(\varphi(t)) \geq f(b)+f^{\prime}(b)(\varphi(t)-b)$.

Integrating the two side of the above inequality, we have

$\int_{0}^{a} f(\varphi(t)) d t \geq \int_{0}^{a} f(b) d t+\int_{0}^{a} f^{\prime}(b)(\varphi(t)-b) d t$

$=a f(b)+f^{\prime}(b) \int_{0}^{a} \varphi(t) d t-a f^{\prime}(b) \cdot b$

$=a f(b)+f^{\prime}(b) \int_{0}^{a} \varphi(t) d t-f^{\prime}(b) \cdot a \cdot \frac{1}{a} \int_{0}^{a}(\varphi(t)) d t$.

$=a f(b)$

So we have proved the inequality

$\frac{1}{a} \int_{0}^{a} f(\varphi(t)) d t \geq f\left(\frac{1}{a} \int_{0}^{a} \varphi(t) d t\right)$.

Example 2 (see [3]) Let $a>0, b>0$, prove the following inequality

$\sqrt{a b} \leq \frac{1}{e}\left(\frac{b^{b}}{a^{a}}\right)^{\frac{1}{b-a}} \leq \frac{a+b}{2}$.

Proof. Let $f(x)=-\ln x$, then $f^{\prime \prime}(x)=\frac{1}{x^{2}}>0, x \in(0,+\infty)$. By Lemma 1 , we see that $f(x)$ is a convex function on $(0,+\infty)$. By hadamard inequality (5) in Lemma 3, for two positive real numbers $a, b$, we have

$$
\frac{\ln b+\ln a}{2} \leq \frac{\int_{a}^{b} \ln x d x}{b-a} \leq \ln \frac{a+b}{2},
$$

i.e.

$$
\ln \sqrt{a b} \leq \ln \left(\frac{1}{e}\left(\frac{b^{b}}{a^{a}}\right)^{\frac{1}{b-a}}\right) \leq \ln \frac{a+b}{2} .
$$

It implies that

$$
\sqrt{a b} \leq \frac{1}{e}\left(\frac{b^{b}}{a^{a}}\right)^{\frac{1}{b-a}} \leq \frac{a+b}{2} .
$$


Example 3 (see [4]) Let $x \in\left(0, \frac{\pi}{2}\right)$, prove the inequality

$(\sin x)^{1-\cos 2 x}+(\cos x)^{1+\cos 2 x} \geq \sqrt{2}$.

Proof. Since $f(x)=x^{x}$ is a convex function on $(0,+\infty)$, for $a>0, b>0$, we have $f\left(\frac{a+b}{2}\right) \leq \frac{1}{2}[f(a)+f(b)]$.

Let $a=\sin ^{2} x, b=\cos ^{2} x$, then

$f\left(\frac{a+b}{2}\right)=f\left(\frac{\sin ^{2} x+\cos ^{2} x}{2}\right)=f\left(\frac{1}{2}\right)=\left(\frac{1}{2}\right)^{\frac{1}{2}}=\frac{\sqrt{2}}{2}$.

Since $\frac{1}{2}[f(a)+f(b)]=\frac{1}{2}\left[\left(\sin ^{2} x\right)^{\sin ^{2} x}+\left(\cos ^{2} x\right)^{\cos ^{2} x}\right]$, we have

$\left(\sin ^{2} x\right)^{\sin ^{2} x}+\left(\cos ^{2} x\right)^{\cos ^{2} x} \geq \sqrt{2}$.

It implies that

$(\sin x)^{1-\cos 2 x}+(\cos x)^{1+\cos 2 x} \geq \sqrt{2}$.

Example 4 (see [5]) Suppose that $f(x)$ is a convex function on $[N,+\infty)$, and $f^{\prime}(+\infty)$ is bounded, then $\sum_{i=1}^{\infty} a_{k}$ converges, where $a_{k}=\frac{f(k)+f(k+1)}{2}-\int_{k}^{k+1} f(x) d x$.

Proof. Since $f(x)$ is a convex function on $[N,+\infty)$, by Lemma 2, we see that $f^{\prime}(x)$ is increasing on $[N,+\infty)$ and $f(x) \geq f(k)+f^{\prime}(k)(x-k)$ holds. So

$a_{k}=\frac{1}{2}\left(f(k)+f(k+1)-2 \int_{k}^{k+1} f(x) d x\right)$

$\leq \frac{1}{2}\left(f(k)+f(k+1)-2 \int_{k}^{k+1}\left[f(k)+(x-k) f^{\prime}(k)\right] d x\right)$

$=\frac{1}{2}\left(f(k)+f(k+1)-2 f(k)-(2 k+1) f^{\prime}(k)+2 k f^{\prime}(k)\right)$

$=\frac{1}{2}\left(f(k+1)-f(k)-f^{\prime}(k)\right)=\frac{1}{2}\left(f^{\prime}(\xi)-f^{\prime}(k)\right)$

$\leq \frac{1}{2}\left(f^{\prime}(k+1)-f^{\prime}(k)\right)$,

where $\xi \in(k, k+1)$. By Lemma 3 we see that $\frac{f(k)+f(k+1)}{2} \geq \int_{k}^{k+1} f(x) d x$ holds, So $\sum_{i=1}^{\infty} a_{k}$ is series with positive term. Since $\sum_{k=n}^{n} a_{k} \leq \frac{1}{2}\left(f^{\prime}(n+1)-f^{\prime}(N)\right) \leq \frac{1}{2}\left(f^{\prime}(+\infty)-f^{\prime}(N)\right)$, we see that positive term series $\sum_{i=1}^{\infty} a_{k}$ converges.

Example 5 (see [5]) Suppose that $f(x)$ is a convex function on $[1,+\infty)$, and $f^{\prime}(+\infty)$ is bounded, then 
the convergence of series $\sum_{k=1}^{\infty} f(k)$ is same as integral $\int_{1}^{+\infty} f(x) d x$.

Proof. Since $f(x)$ is a convex function, by Lemma 2, we know that $f^{\prime \prime}(x) \geq 0$ and $f^{\prime}(x)$ is increasing, by Lemma 3 we see that $\frac{f(k)+f(k+1)}{2} \geq \int_{k}^{k+1} f(x) d x$ holds. By Example 4, we see that the series with positive term

$$
\sum_{k=1}^{\infty}\left(\frac{f(k)+f(k+1)}{2}-\int_{k}^{k+1} f(x) d x\right)
$$

converges. Since

$$
\sum_{k=1}^{\infty}\left(\frac{f(k)+f(k+1)}{2}\right)-\int_{1}^{+\infty} f(x) d x=\sum_{k=1}^{\infty}\left(\frac{f(k)+f(k+1)}{2}-\int_{k}^{k+1} f(x) d x\right),
$$

We see that the convergence of series $\sum_{k=1}^{\infty} \frac{f(k)+f(k+1)}{2}$ is same as the integral $\int_{1}^{+\infty} f(x) d x$. Because $f(x)$ is a convex function, we know that there is a $M>0$ such that $f(x)$ is Invariant sign when $x>M$. Thus, the convergence of series $\sum_{k=1}^{\infty} \frac{f(k)+f(k+1)}{2}$ is same as $\sum_{k=1}^{\infty} f(k)$. In summary, the convergence of series $\sum_{k=1}^{\infty} f(k)$ is same as $\int_{1}^{+\infty} f(x) d x$.

\section{Summary}

In many problems, we often encountered in the proof of some inequalities. Proof of inequality often requires some high skills. Proof process can be made to be simple and clear using properties of a convex function. But the key of this method is constructing appropriate convex functions. This paper discusses applications of convex function in proving inequalities and verifying astringency of series.

\section{Acknowledgement}

This research was supported by Guangxi Natural Science Foundation (Project No. 2012GXNS FAA053009) and Scientific Research Foundation of the Education Department of Guangxi Autonomous Region of China (No. KY2015ZD103), and the high school specialty and curriculum integration project of Guangxi Zhuang Autonomous Region (No.GXTSZY2220).

\section{References}

[1] Department of mathematics, East China Normal University, Mathematical analysis, third ed., Higher Education Press, Beijing, 2001.

[2] L. wang, On Some Extentions of Hadamard Inequalities for Convex Functions, Mathematics in practice and theory,32(6)(2002)1027-1030.

[3] Q. Mao, Analysis of two important inequalities of convex, Journal of Huangshi Institute of Technology,24(5)(2008)47-49.

[4] Y. Shang and S. You, Convex function and its application in proving inequalities, Journal of Guangzhou University (Natural Science Edition),4(1)(2005)1-6. 
[5] M. Lin, Proof of series convergence using properties of convex function, Journal of Wuzhou teachers college of Guangxi, 19(2)(2003) 61-63.

[6] S.S. Dragomir, S. Fitzpatrik, The Hadamard's inequality for s-convex functions in the second sense, Demonstration Math. 32 (4) (1999) 687-696.

[7] M. Alomari and M. Darus, The Hadamard's inequality for s-convex function of 2-variables On The co-ordinates, Int. Journal of Math. Analysis. 2 (13) (2008) 629-638.

[8] M.Z. Sarikaya, A. Saglam, H. Yildirim, On some Hadamard-type inequalities for h-convex functions, J. Math. Inequalities. 2 (2008) 335-341.

[9] B. Xi, F. Qi, Some inequalities of Hermite-Hadamard type for h-convex functions, Adv. Inequal. Appl. 2 (1) (2013) 1-15. 